\title{
Common Coupled Fixed Point Theorems for Weakly F-contractive Mappings in Topological Spaces
}

\author{
Savita Rathee ${ }^{1}$, Priyanka Gupta ${ }^{2, *}$ \\ ${ }^{1}$ Department of Mathematics, Maharshi Dayanand University, Rohtak-124001, Haryana, India \\ ${ }^{2}$ Department of Mathematics, Pt. Chiranji Lal Sharma Govt. P.G. College, Karnal-132001, Haryana, India
}

Received May 5, 2020; Revised June 15, 2020; Accepted June 28, 2020

Copyright $@ 2020$ by authors, all rights reserved. Authors agree that this article remains permanently open access under the terms of the Creative Commons Attribution License 4.0 International License

\begin{abstract}
In late sixties, Furi and Vignoli proved fixed point results for $\alpha$-condensing mappings on bounded complete metric spaces. Bugajewski generalized the results to "weakly F-contractive mappings" on topological spaces(TS). Bugajeski and Kasprzak proved several fixed point results for "weakly F-contractive mapping" using the approach of lower(upper) semi-continuous functions. After that, by modifying the concept of "weakly F-contractive mappings", the coupled fixed point results were proved by Cho, Shah and Hussain on topological space. On different spaces, common coupled fixed point results were discussed by Liu, Zhou and damjanovic, Nashine and Shatanawi and many other authors. In this work, we prove the common coupled fixed point theorems by adopting the modified definition of weakly F-contractive mapping $\gamma: T \rightarrow T$, where $T$ is a topological space. After that, we extend the result of Cho, Shah and Hussain for Banach spaces to common coupled quasi solutions enriched with a relevant transitive binary relation. Also, we give an example in the support of proved result. Our results extend and generalize several existing results in the literature.
\end{abstract}

Keywords Topological Space, Weakly F-contractive Mapping, Common Coupled Fixed Point, Relatively Compact Set

\section{Introduction}

As already known that, if $\gamma: T \rightarrow T$ is a "weakly contractive mapping" for a compact metric space $T$, then $\gamma$ has a "fixed point" in $T$ (see [7]). In late sixties, the above concept was extended by Furi and Vignoli ([8]) to $\alpha$-condensing mappings acting on a "bounded complete metric space" which have been generalized by Bugajewski([2]) by make use of the approach of "weakly F-contractive mappings" acting on a TS.

Chen et al.([4]) introduced a more general notion of "lower(upper) semi-continuous" function using KKMmappings which was redefined by Bugajewski ([3]) for weakly F-contractive mappings to devise and demonstrate several fixed point results.

In 2010, Shah et al.([10]) take further the work of Bugajewski ([3]) to common fixed point results adopting the approach of "weakly F-contractive mappings" in TS. As per need of "coupled fixed point" results, Cho et al.([6]) modified the concept of "weakly F-contractive mappings" $\gamma: T \times T \rightarrow T$ on TS $T$.

Further common coupled fixed point results was studied by many authors $([9],[11])$ in partially ordered metric space. By using the semi-order method, Cho et al.([5]) discussed the solvability of "coupled quasi solutions" for the non-linear operator equations.

In this work, we reformulate and prove the outcomes of Cho et al.([6]) for common coupled fixed points by make use of the modified definition of "weakly F-contractive" mappings $\gamma: T \rightarrow T$, where $T$ is a TS. Also, by taking certain iteration of the mapping as weakly $\mathbf{F}$-contractive, we give some common coupled fixed point results. As a corollary, we get a broadening to "common coupled fixed points" of ( [6] , theorem 3) for "Banach spaces" with a quasimodulus enriched with a relevant transitive binary relation.

\section{Preliminaries}

In this section, firstly we recollect the definition of a "lower semi-continuous from above(lsca)" function introduced in [6].

Definition 2.1 ([6]) Let $T$ be a TS. A function $\gamma: T \times T \rightarrow R$ on TS $T$ is forenamed to be lsca at a point $\left(l_{0}, m_{0}\right) \in T \times T$ if, for any net $\left\{\left(l_{t}, m_{t}\right)\right\}_{t \in \Lambda}$ in $T \times T$ converging to $\left(l_{0}, m_{0}\right)$ such that $\gamma\left(l_{t_{1}}, m_{t_{1}}\right) \leq \gamma\left(l_{t_{2}}, m_{t_{2}}\right)$ for any $t_{1}, t_{2} \in \Lambda$ with $t_{2} \leq t_{1}, \gamma\left(l_{0}, m_{0}\right) \leq \lim _{t \in \Lambda} \gamma\left(l_{t}, m_{t}\right)$.

A function $\gamma: T \times T \rightarrow \mathbf{R}$ is forenamed to be lsca if it is lsca $\forall(l, m) \in T \times T$. 
Example 2.2 [6] Let $\gamma: T \times T \rightarrow \mathbf{R}$ where $T=\{(0,0)\} \cup$ $\mathbf{R}^{-} \times \mathbf{R}^{-} \cup \mathbf{R}^{+} \times \mathbf{R}^{+}$outlined by

$$
\gamma(c, d)= \begin{cases}c+d+1, & \text { if } \quad(c, d) \in \mathbf{R}^{+} \times \mathbf{R}^{+} \cup\{(0,0)\} \\ c+d, & \text { if } \quad(c, d) \in \mathbf{R}^{-} \times \mathbf{R}^{-}\end{cases}
$$

Clearly, at $(0,0), \gamma$ is lsca, but it is not "lower semicontinuous" at $(0,0)$

The subsequent lemmas establish some properties of lsca mappings.

Lemma 2.3 [4] Let $\gamma: T \rightarrow R$ be an lsca function on compact TS T. Then $\exists l_{0} \in T$ such that $\gamma\left(l_{0}\right)=\inf \{\gamma(l): l \in$ $T\}$.

Lemma 2.4 [6] Let $\gamma_{1}: T \times T \rightarrow T$ be a continuous function on TS T. If $\gamma_{2}: T \rightarrow R$ is a lsca function, then the composition function $\eta=\gamma_{2} \circ \gamma_{1}: T \times T \rightarrow R$ is also lsca.

Remark 2.5 Let $\gamma: T \times T \rightarrow T$ be a "continuous function" and $F: T \times T \rightarrow R$ be lsca on TS T. Then $\eta: T \times T \rightarrow R$ outlined by $\eta(c, d)=F(c, \gamma(c, d))($ or $\eta(c, d)=F(c, \gamma(d, c)))$ is also lsca. For, let $\left\{\left(c_{t}, d_{t}\right)\right\}_{t \in \Lambda}$ be a net in $T \times T$ converging to a point $(c, d) \in T \times T$. Since $\gamma$ is continuous, we have $\lim _{t \in \Lambda} \gamma\left(c_{t}, d_{t}\right)=\gamma(c, d)$. Suppose that $\eta\left(c_{t_{1}}, d_{t_{1}}\right) \leq$ $\eta\left(c_{t_{2}}, d_{t_{2}}\right)$ if $t_{2} \leq t_{1}$. Because $F$ is lsca, we have $\eta(c, d)=F(c, \gamma(c, d)) \leq \lim _{t \in \Lambda} F\left(c_{t}, \gamma\left(c_{t}, d_{t}\right)\right)=$ $\lim _{t \in \Lambda} \eta\left(c_{t}, d_{t}\right)$.

Definition 2.6 [3] Let $\mathbf{F}: T \times T \rightarrow R$ be lsca on TS T. The mapping $\gamma: T \rightarrow T$ is forenamed to be:

(1) "weakly $\mathbf{F}$-contractive" if $\mathbf{F}(\gamma(c), \gamma(d))$

$\mathbf{F}(c, d) \forall c, d \in T$ with $c \neq d$;

(2) "strongly $\mathbf{F}$-expansive" if $\mathbf{F}(\gamma(c), \gamma(d)) \quad>$

$\mathbf{F}(c, d) \forall c, d \in T$ with $c \neq d$.

Cho et al.([6]) extended the above definition in a TS $T$ to the "product space" $T \times T$.

Definition 2.7 [6] Let $\mathbf{F}: T \times T \rightarrow R$ be lsca on a TS $T$. The mapping $\gamma: T \times T \rightarrow T$ is forenamed to be:

(1) "weakly $\mathbf{F}$-contractive in the first coordinate" if $\mathbf{F}(\gamma(c, d), \gamma(p, q))<\mathbf{F}(c, p) \quad \forall \quad c, d, p, q \in T$ with $(c, d) \neq(p, q)$;

(2) "strongly $\mathbf{F}$-expansive in the first coordinate" if $\mathbf{F}(\gamma(c, d), \gamma(p, q))>\mathbf{F}(c, p) \quad \forall \quad c, d, p, q \in T$ with $(c, d) \neq(p, q)$;

(3) "weakly $\mathbf{F}$-contractive in the second coordinate" if $\mathbf{F}(\gamma(c, d), \gamma(p, q))<\mathbf{F}(d, q) \quad \forall \quad c, d, p, q \in T$ with $(c, d) \neq(p, q)$;

(4) "strongly $\mathbf{F}$-expansive in the second coordinate" if $\mathbf{F}(\gamma(c, d), \gamma(p, q))>\mathbf{F}(d, q) \quad \forall \quad c, d, p, q \in T$ with $(c, d) \neq(p, q)$;

(5) "weakly contractive" if $\mathbf{F}(\gamma(c, d), \gamma(p, q))<$ $\min \{\mathbf{F}(c, p), \mathbf{F}(d, q)\} \forall c, d, p, q \in T$ with $(c, d) \neq(p, q)$;

(6) "strongly expansive" if $\mathbf{F}(\gamma(c, d), \gamma(p, q))>$ $\max \{\mathbf{F}(c, p), \mathbf{F}(d, q)\} \forall c, d, p, q \in T$ with $(c, d) \neq(p, q)$.
Definition 2.8 [9] (1) An element $(c, d) \in T \times T$ is called a "coupled fixed point" of the mapping $\gamma: T \times T \rightarrow T$ on $T S$ $T$ if $\gamma(c, d)=c$ and $\gamma(d, c)=d$.

(2) An element $(c, d) \in T \times T$ is called a "coupled coincidence point" of the mapping $\gamma: T \times T \rightarrow T$ and $\eta: T \rightarrow T$ if $\gamma(c, d)=\eta(c)$ and $\gamma(d, c)=\eta(d)$.

(3) An element $(c, d) \in T \times T$ on TS $T$ is called a "common coupled fixed point" of the mapping $\gamma: T \times T \rightarrow T$ and $\eta: T \rightarrow T$ if $\gamma(c, d)=\eta(c)=c$ and $\gamma(d, c)=\eta(d)=d$.

(4) Let $T$ be a TS. The mappings $\gamma: T \times T \rightarrow T$ and $\eta:$ $T \rightarrow T$ are commuting if $\eta(\gamma(c, d))=\gamma(\eta(c), \eta(d)) \forall c, d \in$ $T$.

\section{Main Results}

In this section, we enhance the results in [6] to the setting of two mappings having a exactly one "common coupled fixed point". Before this, we define the following terms which we will use in our theorem.

Let us outline the two sets $G=\left\{l_{n}: n \geq 1\right\}$ and $H=$ $\left\{m_{n}: n \geq 1\right\}$ where $l_{n}, m_{n}$ are in any non-empty set T. Now define the sets $\triangle_{G \times H}=\left\{\left(l_{n}, m_{n}\right): n \geq 1\right\}$ and $\triangle_{H \times G}=$ $\left\{\left(m_{n}, l_{n}\right): n \geq 1\right\}$ where $\triangle_{G \times H} \subseteq G \times H$ and $\triangle_{H \times G} \subseteq$ $H \times G$. Similarly $\bar{\triangle}_{G \times H} \subseteq \bar{G} \times \overline{\bar{H}}$ and $\bar{\triangle}_{H \times G} \subseteq \bar{H} \times \overline{\bar{G}}$ where $\bar{G}$ denotes the closure of $G$ and so on.

Theorem 3.1 Let $\gamma: T \times T \rightarrow T$ and $\eta: T \rightarrow T$ are two commuting mappings on a TS T such that for each countable sets $E, F \subseteq T$,

$E=\gamma\left(\triangle_{E \times F}\right) \cup\left\{\eta\left(l_{0}\right)\right\}$ and $F=\gamma\left(\triangle_{F \times E}\right) \cup\left\{\eta\left(m_{0}\right)\right\} \Longrightarrow$ $E, F$ are relatively compact

where $\triangle_{E \times F} \subseteq E \times F, \triangle_{F \times E} \subseteq F \times E$ and $l_{0}, m_{0} \in T$. If

(i) $\gamma$ is "weakly $\mathbf{F}$-contractive" and continuous or

(ii) $\eta$ is "weakly $\mathbf{F}$-contractive" and continuous with $\eta(E) \subseteq$ $E, \eta(F) \subseteq F$,

then $\gamma$ and $\eta$ have exactly one "common coupled fixed point".

proof. Let $l_{1}=\eta\left(l_{0}\right), m_{1}=\eta\left(m_{0}\right)$ and outline the sequences $\left\{l_{n}\right\}_{n \geq 1}$ and $\left\{m_{n}\right\}_{n \geq 1}$ in $T$ in such a way: $l_{n+1}=\gamma\left(l_{n}, m_{n}\right)$, $m_{n+1}=\gamma\left(m_{n}, l_{n}\right)$. Outline the sets

$G=\left\{l_{n}: n \geq 1\right\}$ and $H=\left\{m_{n}: n \geq 1\right\}$. Then, we have

$$
\begin{gathered}
G=\left\{l_{n+1}: n \geq 1\right\} \cup\left\{\eta\left(l_{0}\right)\right\} \\
G=\left\{\gamma\left(l_{n}, m_{n}\right): n \geq 1\right\} \cup\left\{\eta\left(l_{0}\right)\right\} \\
G=\gamma\left(\triangle_{G \times H}\right) \cup\left\{\eta\left(l_{0}\right)\right\}
\end{gathered}
$$

Similarly,

$$
H=\gamma\left(\triangle_{H \times G}\right) \cup\left\{\eta\left(m_{0}\right)\right\}
$$

Therefore, by supposition, $G$ and $H$ are relatively compact.

Let $W=(\bar{G} \times \bar{H}) \cup(\bar{H} \times \bar{G})$ and $W_{\triangle}=\left(\bar{\triangle}_{G \times H}\right) \cup\left(\bar{\triangle}_{H \times G}\right)$. Then $W$ and $W_{\triangle}$ are compact and contained in $T \times T$ with $W_{\triangle}$ contained in $W$.

Outline $\phi: W_{\triangle} \longrightarrow R$ by

$$
\phi(l, m)= \begin{cases}\mathbf{F}(l, \gamma(l, m)), & \text { if } \gamma \text { is continuous } \\ \mathbf{F}(l, \eta(l)), & \text { if } \eta \text { is continuous }\end{cases}
$$


for all $(l, m) \in W_{\triangle}$.

Since $\mathbf{F}$ is lsca so for continuity of $\gamma$ or $\eta$ mappings, $\phi$ is lsca by using Remark (2.5) and hence $\phi$ has a minimum, say at $(c, d) \in W_{\triangle}$ by Lemma (2.3). Therefore, either $(c, d) \in \bar{\triangle}_{G \times H}$ or $(c, d) \in \bar{\triangle}_{H \times G}$.

Case-1 Suppose that $(c, d) \in \bar{\triangle}_{G \times H}$ and $\gamma$ is continuous and weakly F-contractive. Then $(d, c) \in \bar{\triangle}_{H \times G}$. Hence $\exists$ two sequences $\left\{\left(c_{n}, d_{n}\right)\right\} \subseteq \triangle_{G \times H}$ and $\left\{\left(d_{n}, c_{n}\right)\right\} \subseteq \triangle_{H \times G}$ such that $\lim _{n \rightarrow \infty}\left(c_{n}, d_{n}\right)=(c, d)$ and $\lim _{n \rightarrow \infty}\left(\bar{d}_{n}, c_{n}\right)=$ $(d, c)$.

Due to the continuity of $\gamma$, we have

$\lim _{n \rightarrow \infty} \gamma\left(c_{n}, d_{n}\right)=\gamma(c, d)$ and $\lim _{n \rightarrow \infty} \gamma\left(d_{n}, c_{n}\right)=$ $\gamma(d, c)$

But $\left(\gamma\left(c_{n}, d_{n}\right), \gamma\left(d_{n}, c_{n}\right)\right)=\left(c_{n+1}, d_{n+1}\right) \in \triangle_{G \times H}$.

Now, we proclaim that $(\gamma(c, d), \gamma(d, c))=(c, d)$. Suppose that $(\gamma(c, d), \gamma(d, c)) \neq(c, d)$. Since $\gamma$ is weakly $\mathbf{F}$-contractive, we have

$$
\begin{aligned}
\phi(\gamma(c, d), \gamma(d, c)) & =\mathbf{F}(\gamma(c, d), \gamma(\gamma(c, d), \gamma(d, c))) \\
& <\min \{\mathbf{F}(c, \gamma(c, d)), \mathbf{F}(d, \gamma(d, c))\} \\
& =\min \{\phi(c, d), \phi(d, c)\} \\
& =\phi(c, d),
\end{aligned}
$$

which is contravention to the minimality of $\phi$ at $(c, d)$. Hence $\gamma(c, d)=c$ and $\gamma(d, c)=d$.

One can easily see that $\eta(c)=c$ and $\eta(d)=d$.

Undoubtedly, if $\eta(c) \neq c$ and $\eta(d) \neq d$, then we have

$\mathbf{F}(c, \eta(c))=\mathbf{F}(\gamma(c, d), \eta(\gamma(c, d)))$

$\mathbf{F}(\gamma(c, d), \gamma(\eta c, \eta c))<\mathbf{F}(c, \eta c)$, (because $\gamma$ and $\eta$ are commuting) which is contravention.

Similarly,

$\mathbf{F}(d, \eta(d)) \quad=\quad \mathbf{F}(\gamma(d, c), \eta(\gamma(d, c)))=$ $\mathbf{F}(\gamma(d, c), \gamma(\eta d, \eta c))<\mathbf{F}(d, \eta d)$, (because $\gamma$ and $\eta$ are commuting) which is contravention. Thus $\eta(c)=c, \eta(d)=d$.

Case-2 Suppose that $(c, d) \in \bar{\triangle}_{H \times G}$ and $\gamma$ is continuous and weekly F-contractive. Then $(d, c) \in \bar{\triangle}_{G \times H}$. Pursuing the proof of case-1, we can also wind up that $\gamma(c, d)=c, \gamma(d, c)=q$ and $\eta(c)=c, \eta(d)=d$.

(ii) Case-1: Suppose that $(c, d) \in \bar{\triangle}_{G \times H}$ and $\eta$ is continuous and weekly $\mathbf{F}$-contractive with $\eta(E) \subseteq E, \eta(F) \subseteq F$. Then $\phi(l, m)=\mathbf{F}(l, \eta l)$ as $\eta$ is continuous. Put $E=G$ and $F=H$ then $c \in \bar{G}, d \in \bar{H}, g$ is continuous and $\eta(G) \subseteq G, \eta(H) \subseteq H$ signifies that $\eta(c) \in \bar{G}, \eta(d) \in \bar{H}$.

Now, we assert that $\eta(c)=c, \eta(d)=d$, for otherwise, we will have

$$
\phi(\eta c, \eta d)=\mathbf{F}(\eta c, \eta \eta c)<\mathbf{F}(c, \eta c)=\phi(c, d)
$$

which is contradiction to the minimality of $\phi$ at $(c, d)$. Hence $\eta(c)=c$ and $\eta(d)=d$. Now, suppose that $\gamma(c, d) \neq c$ and $\gamma(d, c) \neq d$, then we have

$\mathbf{F}(d, \gamma(c, d))=\mathbf{F}(\eta c, \gamma(\eta c, \eta d))=\mathbf{F}(\eta c, \eta(\gamma(c, d)))<$ $\mathbf{F}(c, \gamma(c, d))$ (because $\gamma$ and $\eta$ are commuting) which is contravention. Hence $\gamma(c, d)=c$.
Similarly,

$$
\begin{aligned}
\mathbf{F}(d, \gamma(d, c)) & =\mathbf{F}(\eta d, \gamma(\eta d, \eta c)) \\
& =\mathbf{F}(\eta d, \eta(\gamma(d, c))) \\
& <\mathbf{F}(q, \gamma(d, c))
\end{aligned}
$$

which is contravention. Hence $\gamma(d, c)=q$

Thus $\gamma$ and $\eta$ have a "common coupled fixed point".

Case-2: Suppose that $(c, d) \in \bar{\triangle}_{H \times G}$ and $\eta$ is continuous and weakly F-contractive with $\eta(E) \subseteq E$ and $\eta(F) \subseteq F \Longrightarrow$ $(d, c) \in \bar{\triangle}_{G \times H}$ means $c \in \bar{H}$ and $\bar{d} \in \bar{G}$. Then $\phi(l, m)=$ $F(l, \eta l)$ as $\eta$ is continuous. Put $E=G$ and $F=H$. Then $d \in \bar{G}, c \in \bar{H}, \eta$ is continuous and $\eta(G) \subseteq G, \eta(H) \subseteq H$ implies that $\eta(d) \in \bar{G}, \eta(c) \in \bar{H}$. Pursuing the proof of case1 , we can also wind up that $\gamma(c, d)=c, \gamma(d, c)=d$ and $\eta(c)=c, \eta(d)=d$.

Uniqueness, suppose $\exists(r, s) \in W_{\triangle}$ s.t. $\gamma(r, s)=\eta(r)=r$ and $\gamma(s, r)=\eta(s)=s$.

Then, we have

$\mathbf{F}(c, r)=\mathbf{F}(\gamma(c, d), \gamma(r, s))<\mathbf{F}(c, r)$

$\mathbf{F}(q, s)=\mathbf{F}(\gamma(d, c), \gamma(s, r))<\mathbf{F}(d, s)$

$\mathbf{F}(c, d)=\mathbf{F}(\eta c, \eta d)<\mathbf{F}(c, d)$ (because $\eta$ is weakly $\mathbf{F}$ contractive)

which is not true. Hence $\gamma$ and $\eta$ have exactly one common coupled fixed point.

If $\eta=i d_{T}$, then above theorem dwindle to [[6],Theorem 3.1]

Corollary 3.2 [6] Let $\gamma: T \times T \rightarrow T$ be a "continuous and weakly $\mathbf{F}$-contractive" mappings such that for each countable sets $E, F \subseteq T$,

$E=\gamma\left(\triangle_{E \times F}\right) \cup\left\{l_{0}\right\}$ and $F=\gamma\left(\triangle_{F \times E}\right) \cup\left\{m_{0}\right\} \Longrightarrow E, F$ are relatively compact,

where $\triangle_{E \times F} \subseteq E \times F, \quad \triangle_{F \times E} \subseteq F \times E$ and $l_{0}, m_{0} \in T$, then $\gamma$ has a "coupled fixed point" in $T \times T$.

Corollary 3.3 Let $\gamma: T \times T \rightarrow T$ and $\eta: T \rightarrow T$ be two commuting mappings on a metric space $(T, d)$ such that for each countable sets $E, F \subseteq T$,

$E=\gamma\left(\triangle_{E \times F}\right) \cup\left\{\eta\left(l_{0}\right)\right\}$ and $F=\gamma\left(\triangle_{F \times E}\right) \cup\left\{\eta\left(m_{0}\right)\right\} \Longrightarrow$ $E, F$ are relatively compact

where $\triangle_{E \times F} \subseteq E \times F, \triangle_{F \times E} \subseteq F \times E$ and $l_{0}, m_{0} \in T$. If

(i) $\gamma$ is "weakly contractive" and continuous or

(ii) $\eta$ is "weakly contractive" and continuous with $\eta(E) \subseteq$ $E, \eta(F) \subseteq F$,

then $\gamma$ and $\eta$ have exactly one common coupled fixed point.

proof. Taking $\mathbf{F}=d$ in above Theorem, we get desired result.

Corollary 3.4 Let $\gamma: T \times T \rightarrow T$ and $\eta: T \rightarrow T$ be two commuting mappings on a compact TS $T$ such that for each countable sets $E, F \subseteq T$,

$E=\gamma\left(\triangle_{E \times F}\right) \cup\left\{\eta\left(l_{0}\right)\right\}$ and $F=\gamma\left(\triangle_{F \times E}\right) \cup\left\{\eta\left(m_{0}\right)\right\} \Longrightarrow$ $E, F$ are closed,

where $\triangle_{E \times F} \subseteq E \times F, \triangle_{F \times E} \subseteq F \times E$ and $l_{0}, m_{0} \in T$. If

(i) $\gamma$ is "weakly contractive" and continuous or

(ii) $\eta$ is "weakly $\mathbf{F}$-contractive" and continuous with $\eta(E) \subseteq$ $E, \eta(F) \subseteq F$,

then $\gamma$ and $\eta$ have exactly one common coupled fixed point.

proof. We get the desired result from Theorem (3.1). 
Theorem 3.5 Let $h: T \rightarrow T, r: T \times T \rightarrow T$ and $\eta: T \rightarrow T$ be mappings on a TS T. Set $\gamma^{k}=h^{k} \circ r$ and suppose that $\exists$ $l_{0}, m_{0} \in T$ such that for each countable sets $E, F \subseteq T$,

(i) $E=\gamma^{k}\left(\triangle_{E \times F}\right) \cup\left\{\eta\left(l_{0}\right)\right\}$ and $F=\gamma^{k}\left(\triangle_{F \times E}\right) \cup$ $\left\{\eta\left(m_{0}\right)\right\} \Longrightarrow E, F$ are relatively compact,

(ii) $E=\gamma^{k}\left(\triangle_{E \times F}\right) \cup\left\{\eta^{k}\left(l_{0}\right)\right\}$ and $F=\gamma^{k}\left(\triangle_{F \times E}\right) \cup$ $\left\{\eta^{k}\left(m_{0}\right)\right\} \Longrightarrow E, F$ are relatively compact

where $\triangle_{E \times F} \subseteq E \times F, \triangle_{F \times E} \subseteq F \times E$ and $\gamma^{k}$, $\eta$ commute on T. If

(i) $h$ and $r$ are continuous and $h^{k}, r$ are "weakly $\mathbf{F}$ contractive" or

(ii) $\eta$ is continuous and $\eta^{k}$ is "weakly F-contractive" with $\eta(E) \subseteq E, \quad \eta(F) \subseteq F$,

then $\gamma$ and $\eta$ have exactly one common coupled fixed point in $T \times T$.

proof. Set $\gamma^{k}=h^{k} \circ r$. Let $l_{1}=\eta^{k}\left(l_{0}\right), m_{1}=\eta^{k}\left(m_{0}\right)$ for any $k \in \mathbf{N}$ and outline the sequences $\left\{l_{n}\right\}_{n \geq 1}$ and $\left\{m_{n}\right\}_{n \geq 1}$ in $T$ in such a way: $l_{n+1}=\gamma_{k}\left(l_{n}, m_{n}\right), m_{n+1}=\gamma^{k}\left(m_{n}, l_{n}\right)$ for each $n \geq 1$. Outline the sets

$G=\left\{l_{n}: n \geq 1\right\}$ and $H=\left\{m_{n}: n \geq 1\right\}$. Then, we have

$$
G=\gamma^{k}\left(\triangle_{G \times H}\right) \cup\left\{\eta^{k}\left(l_{0}\right)\right\}
$$

where, $\triangle_{G \times H}=\left\{\left(l_{n}, m_{n}\right): n \geq 1\right\}$.

Similarly,

$$
H=\gamma^{k}\left(\triangle_{H \times G}\right) \cup\left\{\eta^{k}\left(m_{0}\right)\right\}
$$

where, $\triangle_{H \times G}=\left\{\left(m_{n}, l_{n}\right): n \geq 1\right\}$.

And so, by assumption, $G$ and $Q$ are relatively compact.

Let $W=(\bar{G} \times \bar{H}) \cup(\bar{H} \times \bar{G})$ and $W_{\triangle}=\left(\bar{\triangle}_{G \times H}\right) \cup\left(\bar{\triangle}_{H \times G}\right)$. Then $W$ and $W_{\triangle}$ are compact and conained in $T \times T$ with $W_{\triangle}$ contained in $W$.

Define $\phi: W_{\triangle} \longrightarrow R$ by

$$
\phi(l, m)= \begin{cases}\mathbf{F}\left(l, \gamma^{k}(l, m)\right), & \text { if } \gamma \text { is continous } \\ \mathbf{F}\left(l, \eta^{k} l\right), & \text { if } \eta \text { is continous. }\end{cases}
$$

Since $\mathbf{F}$ is lsca so for continuity of $\gamma$ of $\eta$ mappings, $\phi$ is lsca by using Remark (2.5) and hence, say at $(c, d) \in W_{\triangle}$, $\phi$ has a minimum, by Lemma (2.3). Therefore, either $(c, d) \in \bar{\triangle}_{G \times H}$ or $(c, d) \in \bar{\triangle}_{H \times G}$.

Case-1: Assume that $(c, d) \in \bar{\triangle}_{G \times H}$ and $h^{k}, r$ are continuous and "weakly $\mathbf{F}$-contractive", it pursue that $\gamma^{k}$ is continuous and "weakly $\mathbf{F}$-contractive". Then $(d, c) \in \bar{\triangle}_{H \times G}$. Hence $\exists$ two sequences $\left\{\left(c_{n}, d_{n}\right)\right\} \subseteq \triangle_{G \times H}$ and $\left\{\left(d_{n}, c_{n}\right)\right\} \subseteq \triangle_{H \times G}$ such that $\lim _{n \rightarrow \infty}\left(c_{n}, d_{n}\right)=(c, d)$ and $\lim _{n \rightarrow \infty}\left(d_{n}, c_{n}\right)=$ $(d, c)$.

Since $\gamma^{k}$ is continuous, we have

$\lim _{n \rightarrow \infty} \gamma^{k}\left(c_{n}, d_{n}\right)=\gamma^{k}(c, d)$ and $\lim _{n \rightarrow \infty} \gamma^{k}\left(d_{n}, c_{n}\right)=$ $\gamma^{k}(d, c)$

$\operatorname{But}\left(\gamma^{k}\left(c_{n}, d_{n}\right), \gamma^{k}\left(d_{n}, c_{n}\right)\right)=\left(c_{n+1}, d_{n+1}\right) \in \triangle_{G \times H}$.

which signifies that $\left(\gamma^{k}(c, d), \gamma^{k}(d, c)\right) \in \bar{\triangle}_{G \times H}$.

Now, we proclaim that $\left(\gamma^{k}(c, d), \gamma^{k}(d, c)\right)=(c, d)$. Suppose that $\left(\gamma^{k}(c, d), \gamma^{k}(d, c)\right) \neq(c, d)$. Since $\gamma^{k}$ is weakly $\mathbf{F}$ - contractive, we have

$$
\begin{aligned}
\phi\left(\gamma^{k}(c, d), \gamma^{k}(d, c)\right) & =\mathbf{F}\left(\gamma^{k}(c, d), \gamma^{k}\left(\gamma^{k}(c, d), \gamma^{k}(d, c)\right)\right) \\
& <\min \left\{\mathbf{F}\left(c, \gamma^{k}(c, d)\right), \mathbf{F}\left(d, \gamma^{k}(d, c)\right)\right\} \\
& =\min \{\phi(c, d), \phi(d, c)\} \\
& =\phi(c, d),
\end{aligned}
$$

which is contravention to the minimality of $\phi \quad$ at $\quad(c, d)$.

Hence $\left(\gamma^{k}(c, d), \gamma_{k}(d, c)\right)=(c, d)$.

One can easily identify that $\eta^{k}(c)=c$ and $\left.\eta^{k} q\right)=q$.

Suppose that $\eta^{k}(c) \neq c$ and $\eta^{k}(d) \neq d$, then we have $\mathbf{F}\left(c, \eta^{k}(c)\right)=\mathbf{F}\left(\gamma^{k}(c, d), \eta^{k}\left(\gamma^{k}(c, d)\right)\right)=$ $\mathbf{F}\left(\gamma^{k}(c, d), \gamma^{k}\left(\eta^{k} c, \eta^{k} d\right)\right)<\mathbf{F}\left(c, \eta^{k} c\right)$, (because $\gamma$ and $\eta$ are commuting) which is contravention.

Similarly,

$\mathbf{F}\left(d, \eta^{k}(d)\right)=\mathbf{F}\left(\gamma^{k}(d, c), \eta^{k}\left(\gamma^{k}(d, c)\right)\right)=$ $\mathbf{F}\left(\gamma^{k}(d, c), \gamma^{k}\left(\eta^{k} d, \eta^{k} c\right)\right)<\mathbf{F}\left(d, \eta^{k} d\right)$, (because $\gamma$ and $\eta$ are commuting) which is contravention. Thus, $\left(\eta^{k} c, \eta^{k} d\right)=(c, d)$.

Thus, $(c, d)$ is common coupled fixed point of $\gamma^{k}, \eta^{k}$ and hence of $\gamma$ and $\eta$.

Case-2: Suppose that $(c, d) \in \bar{\triangle}_{B \times A}$ and $h^{k}, r$ are continuous and weekly $\mathbf{F}$-contractive. Then $(d, c) \in \bar{\triangle}_{A \times B}$. Pursuing the proof of case-1, we can wind up that $\gamma(c, d)=c, \gamma(d, c)=d$ and $\eta(c)=c, \quad \eta(d)=d$.

(ii) Case-1: Suppose that $(c, d) \in \bar{\triangle}_{G \times H}$ and $\eta_{k}$ is continuous and weekly $\mathbf{F}$-contractive with $\eta(E) \subseteq E, \eta(F) \subseteq F$. Then $\phi(l, m)=F\left(l, \eta^{k} l\right)$ as $\eta$ is continuous. Put $E=G$ and $F=H$ then $c \in \bar{G}, \quad d \in \bar{H}, \eta$ is continuous and $\eta(G) \subseteq G, \eta(H) \subseteq H$ implies that $\eta^{k} c \in \bar{G}, \eta^{k} d \in \bar{H}$.

Now, we claim that $\eta^{k} c=c, \eta^{k} d=d$, for otherwise, we will have

$$
\phi\left(\eta^{k} c, \eta^{k} d\right)=\mathbf{F}\left(\eta^{k} c, \eta^{k} \eta^{k} c\right)<\mathbf{F}\left(c, \eta^{k} c\right)=\phi(c, d)
$$

which is contravention to the minimality of $\phi$ at $(c, d)$. Hence $\eta^{k} c=c$ and $\eta^{k} d=d$. Now, assume that $\gamma^{k}(c, d) \neq c$ and $\gamma^{k}(d, c) \neq d$, then we have

$\mathbf{F}\left(c, \gamma^{k}(c, d)\right)=\mathbf{F}\left(\eta^{k} c, \gamma^{k}\left(\eta^{k} c, \eta^{k} d\right)\right)=$ $\mathbf{F}\left(\eta^{k} c, \eta^{k}\left(\gamma^{k}(c, d)\right)\right)<\mathbf{F}\left(c, \gamma^{k}(c, d)\right)$ (because $\gamma^{k}$ and $\eta$ are commuting) which is contravention. Hence $\gamma^{k}(c, d)=c$. Similarly,

$\mathbf{F}\left(q, \gamma^{k}(d, c)\right)=\mathbf{F}\left(\eta^{k} d, \gamma^{k}\left(\eta^{k} d, \eta^{k} c\right)\right)=$ $\mathbf{F}\left(\eta^{k} d, \eta^{k}\left(\gamma^{k}(d, c)\right)\right)<\mathbf{F}\left(d, \gamma^{k}(d, c)\right)$ (because $\gamma^{k}$ and $\eta$ are commuting) which is contravention. Hence $\gamma^{k}(d, c)=d$.

Case-2: Suppose that $(c, d) \in \bar{\triangle}_{H \times G}$ and $\eta^{k}$ is continuous and weakly F-contractive with $\eta(E) \subseteq E$ and $\eta(F) \subseteq F$ $\Longrightarrow(d, c) \in \bar{\triangle}_{G \times H}$ means $c \in \overline{\bar{H}}$ and $q \in \bar{G}$. Then $\phi(l, m)=\mathbf{F}\left(l, \eta^{k} l\right)$ as $\eta$ is continuous. Put $E=G$ and $F=H$. Then $d \in \bar{G}, c \in \bar{H}, \eta$ is continuous and $\eta(G) \subseteq G, \eta(H) \subseteq H$ implies that $\eta^{k} q \in \bar{G}, \eta^{k} c \in \bar{H}$. Pursuing the proof of case-1, we can also wind up that $\gamma(c, d)=c, \gamma(d, c)=d$ and $\eta(c)=c, \eta(q)=q$.

Uniqueness, suppose $\exists(r, s) \in W_{\triangle}$ s.t. $\gamma^{k}(r, s)=\eta^{k} r=r$ and $\gamma^{k}(s, r)=\eta^{k} s=s$.

Then, we have

$\mathbf{F}(c, r)=\mathbf{F}\left(\gamma^{k}(c, d), \gamma^{k}(r, s)\right)<\mathbf{F}(c, r)$ 
$\mathbf{F}(d, s)=\mathbf{F}\left(\gamma^{k}(d, c), \gamma^{k}(s, r)\right)<\mathbf{F}(d, s)$

$\mathbf{F}(c, d)=\mathbf{F}\left(\eta^{k} c, \eta^{k} d\right)<\mathbf{F}(c, d)$ (because $\eta^{k}$ is weakly F-contractive)

which is false. Thus, $(c, d)$ is the exactly one common coupled fixed point of $\gamma^{k}, \eta^{k}$ and hence of $\gamma$ and $\eta$.

Remark 3.6 In above theorem, if $h=i d_{T}$ and $k=1$, then $\gamma=r$ and so Theorem 3.5 diminshes to Theorem 3.1. Therefore, Theorem 3.5 generalizes the Theorem 3.1.

If $T$ is taken as a Banach space, then we get a nice follow-up of Theorem 3.5, on the survival of "common coupled fixed point".

Let a "Banach space" $T$ having the transitive binary relation $\preceq$ such that for any $\alpha, \beta \in T$, we have

$$
\min _{\preceq}\{\alpha, \beta\}= \begin{cases}\alpha, & \text { if } \alpha \preceq \beta \\ \beta, & \text { if } \beta \preceq \alpha .\end{cases}
$$

Theorem 3.7 Let a "Banach space" $T$ with the "transitive binary relation" $\preceq$ such that $\forall \quad l, m \in T, \quad l \preceq m$ is outlined by $\|l\| \leq\|m\|$. Assume, further that $B, p, q, \eta: T \rightarrow T$ and $e: T \times T \rightarrow T$ are five mappings complying the subsequent conditions:

(a) $0 \preceq q(l)$ and $\|q(l)\|=\|l\| \quad \forall l \in T$;

(b) the mapping $B$ is linear, bounded and, for any $k \in$ $N,\left\|B^{k} l\right\| \leq\|l\| \quad \forall l \in T$ such that $l \neq 0$ with $0 \preceq l ; ;$

(c) if $0 \preceq l \preceq m$, then $B l \preceq B m$;

If either

(i) $q(p(z)-p(w)) \preceq B q(\eta(z)-\eta(w)) \quad \forall z, w \in T$ and $q(e(l, m)-e(u, v)) \preceq B \min \prec\{q \eta(l-u), q \eta(m-$ $v)\} \quad \forall \quad l, m, u, v \in T$ and $\eta$ is contractive,

or

(ii) $q(\eta(z)-\eta(w)) \preceq B q(p(z)-p(w)) \quad \forall \quad z, w \in$ $T \quad$ and $\min _{\preceq}\{q \eta(l-u), q \eta(m-v)\} \preceq$ $B q(e(l, m)-e(u, v)) \quad \forall \quad l, m, u, v \in T$ and $\gamma$ is contractive,

with $\gamma, \eta$ commuting on $T$.

If $\gamma^{k}=p^{k}$ oe $: T \times T \rightarrow T$ is a composition of the $k^{\text {th }}$ iteration $p^{k}$ and $\eta$ and, further there exists $l_{0}, m_{0} \in T$ such that $E=\gamma^{k}\left(\triangle_{E \times F}\right) \cup\left\{\eta\left(l_{0}\right)\right\}$ and $F=\gamma^{k}\left(\triangle_{F \times E}\right) \cup\left\{\eta\left(m_{0}\right)\right\}$ $\Longrightarrow E, F$ are relatively compact

or $E=\gamma^{k}\left(\triangle_{E \times F}\right) \cup\left\{\eta^{k}\left(l_{0}\right)\right\}$ and $F=\gamma^{k}\left(\triangle_{F \times E}\right) \cup$ $\left\{\eta^{k}\left(m_{0}\right)\right\} \Longrightarrow E, F$ are relatively compact

$\forall$ countable sets $E, F \subseteq T$ where $\triangle_{E \times F} \subseteq E \times F$ and $\triangle_{F \times E} \subseteq F \times E$, then $\gamma$ and $\eta$ have exactly one common coupled fixed point in $T \times T$.

proof. Assume that eqn (3.1) holds with $\gamma$ and $\eta$ commuting on $T$. Then, we get

$$
\begin{aligned}
0 & \preceq q\left(\gamma^{2}(l, m)-\gamma^{2}(u, v)\right) \\
& =q\left(p^{2}\left(e(l, m)-p^{2}(e(u, v))\right)\right. \\
& \preceq B q(p \eta e(l, m)-p \eta e(u, v)) \\
& \preceq B q\left(\eta^{3}(l, m)-\eta^{3}(u, v)\right)
\end{aligned}
$$

$$
\begin{aligned}
F\left(\gamma^{k}(l, m), \gamma^{k}(u, v)\right) & =F\left(p^{k}(e(l, m)), p^{k}(e(u, v))\right) \\
& <F(e(l, m), e(u, v)) \\
& <\min _{\breve{ }}\{F(l, u), F(m, v)\}
\end{aligned}
$$

$$
\begin{aligned}
& 0 \text { } q\left(\gamma^{2}(l, m)-\gamma^{2}(u, v)\right) \\
&=q\left(p^{2}(e(l, m))-p^{2}(e(u, v))\right) \\
& \preceq B[q(\eta(p(e(l, m)))-\eta(p(e(u, v))))] \\
& \preceq B[q(p(\eta(e(l, m)))-p(\eta(e(u, v))))] \\
& \preceq B^{2}\left[q\left(\eta^{2}(e(l, m))-\eta^{2}(e(u, v))\right)\right. \\
&=B^{2} q\left(e\left(\eta^{2} l, \eta^{2} m\right)-e\left(\eta^{2} u, \eta^{2} v\right)\right) \\
& \preceq B^{3} \min _{\preceq}\left\{q \eta\left(\eta^{2} l-\eta^{2} u\right), q\left(\eta^{2} m-\eta^{2} v\right)\right\} \\
&=B^{3} \min _{\preceq}\left\{q \eta^{3}(l-u), q \eta^{3}(m-v)\right\} \\
& \preceq B^{3} \min _{\preceq}\{q \eta(l-u), q \eta(m-v)\}
\end{aligned}
$$

Therefore, beyond $k$-steps for $k \in N$, we get

$$
\begin{aligned}
0 & \preceq q\left(\gamma^{k}(l, m)-\gamma^{k}(u, v)\right) \\
& \preceq B^{k+1} \min _{\preceq}\{q \eta(l-u), q \eta(m-v)\}
\end{aligned}
$$

Hence, it pursues that

$$
\begin{aligned}
\left\|\gamma^{k}(l, m)-\gamma^{k}(u, v)\right\| & =\left\|q\left(\gamma^{k}(l, m)-\gamma^{k}(u, v)\right)\right\| \\
& \leq\left\|B^{k+1} \min _{\preceq}\{q \eta(l-u), q \eta(m-v)\}\right\| \\
& \leq\left\|\min _{\preceq}\{q \eta(l-u), q \eta(m-v)\}\right\| \\
& =\min _{\preceq}\{\|\eta(l-u)\|,\|\eta(m-v)\|\} \\
& \leq \min _{\preceq}\{\|(l-u)\|,\|(m-v)\|\}
\end{aligned}
$$

because $\eta$ is contractive.

So, $\gamma^{k}$ is "weakly contractive". Due to the continuity of $\gamma$, the mappings $\gamma$ and $\eta$ have a exactly one "common coupled fixed point" in $T \times T$.

(b) Assume that eqn (3.2) holds and $\gamma$ is contractive $\forall l, m \in T$ with $\gamma, \eta$ commuting. Now, the proof pursues if we interchange $\gamma, \eta$ in (a) above.

The coming example exhibits Theorem 3.1.

Example 3.8 Let $\left(c_{0},\|\cdot\|_{\infty}\right)$ be a "Banach space" of all "null real sequences". Outline a set $T=\left\{u=\left\{u_{n}\right\} \in c_{0}: u_{n} \in\right.$ $[0,1] \forall n \geq 1\}$. Let $\alpha_{n}, \beta_{n}$ be the sequences in $T$ such that $\alpha_{n}, \beta_{n} \in(0,1)$ for each $n>k$ and $\alpha_{n}, \beta_{n} \rightarrow 0$ as $n \rightarrow \infty$ where $k \in N$. Define the mappings $\gamma: T \times T \rightarrow T$ and $\eta: T \rightarrow T$ by

$\gamma(l, m)=\left\{\gamma_{n}\left(l_{n}, m_{n}\right)\right\}, \quad \eta(l)=\left\{\eta_{n}\left(l_{n}\right)\right\}_{n \geq 1} \quad \forall \quad l=$ $\left\{l_{n}\right\}, m=\left\{m_{n}\right\} \in T$ and $\gamma_{n}:[0,1] \times[0,1] \rightarrow[0,1], \eta_{n}:$ $[0,1] \rightarrow[0,1]$ are s.t. for $1 \leq n \leq k$,

$$
\begin{aligned}
& \left|\gamma_{n}\left(l_{n}, m_{n}\right)-\gamma_{n}\left(s_{n}, t_{n}\right)\right|<\min \left\{\left|l_{n}-s_{n}\right|,\left|m_{n}-t_{n}\right|\right\} \\
& \left|\gamma_{n}\left(m_{n}, l_{n}\right)-\gamma_{n}\left(t_{n}, s_{n}\right)\right|<\min \left\{\left|l_{n}-s_{n}\right|,\left|m_{n}-t_{n}\right|\right\}
\end{aligned}
$$




$$
\eta\left(\left(l_{n}\right)-\eta\left(s_{n}\right)\right)<\frac{\left|l_{n}-s_{n}\right|}{3}
$$

and for each $n>k$,

$\gamma_{n}\left(l_{n}, m_{n}\right)=\alpha_{n} l_{n}, \gamma_{n}\left(m_{n}, l_{n}\right)=\beta_{n} m_{n}, \quad \eta\left(\left(l_{n}\right)=\frac{\alpha_{n} l_{n}}{3}\right.$.

We check the suppositions of Theorem 3.1.

(i) Clearly $\gamma$ and $\eta$ are continuous.

(ii) For $l, m \in T$, we have

$$
\begin{gathered}
\|\gamma(l, m)-\gamma(s, t)\|=\sup _{n \geq 1}\left|\gamma_{n}\left(l_{n}, m_{n}\right)-\gamma_{n}\left(s_{n}, t_{n}\right)\right| \\
\|\eta(l)-\eta(m)\|=\sup _{n \geq 1}\left|\eta_{n}\left(l_{n}\right)-\eta_{n}\left(m_{n}\right)\right|
\end{gathered}
$$

Since the sequences $\left\{\gamma_{n}\left(l_{n}, m_{n}\right)\right\}_{n \geq 1} \quad, \quad\left\{\gamma_{n}\left(s_{n}, t_{n}\right)\right\}_{n \geq 1}$ and $\left\{\eta_{n}\left(l_{n}\right)\right\}_{n \geq 1}$ are null sequences, $\exists N \in \mathbf{N}$ such that

$$
\begin{gathered}
\sup _{n \geq 1}\left|\gamma_{n}\left(l_{n}, m_{n}\right)-\gamma_{n}\left(s_{n}, t_{n}\right)\right|=\left|\gamma_{N}\left(l_{N}, m_{N}\right)-\gamma_{N}\left(s_{N}, t_{N}\right)\right| \\
\sup _{n \geq 1}\left|\eta_{n}\left(l_{n}\right)-\eta\left(m_{n}\right)\right|=\left|\eta_{N}\left(l_{N}\right)-\eta\left(m_{N}\right)\right| .
\end{gathered}
$$

Hence, we have

$$
\begin{aligned}
\|\gamma(l, m)-\gamma(s, t)\| & =\left|\gamma_{N}\left(l_{N}, m_{N}\right)-\gamma_{N}\left(s_{N}, t_{N}\right)\right| \\
& <\min \left\{\left|l_{N}-u_{N}\right|,\left|s_{N}-t_{N}\right|\right\} \\
& =\min \left\{\sup _{n \geq 1}\left|l_{N}-u_{N}\right|, \sup _{n \geq 1}\left|s_{N}-t_{N}\right|\right\} \\
& =\min \left\{\left\|l_{N}-u_{N}\right\|,\left\|s_{N}-t_{N}\right\|\right\}
\end{aligned}
$$

and

$$
\begin{array}{r}
\left\|\eta_{n}\left(l_{n}\right)-\eta_{n}\left(m_{n}\right)\right\| \\
=\left|\eta_{N}\left(l_{N}\right)-\eta_{N}\left(m_{N}\right)\right|<\left|l_{N}-m_{N}\right| \\
=\sup _{n \geq 1}\left|l_{n}-m_{n}\right|=\left\|l_{n}-m_{n}\right\| .
\end{array}
$$

Thus $\gamma$ and $\eta$ are weakly contractive and continuous mappings.Next assume that, for some countable sets $E, M \subseteq T$, we have

$$
\begin{aligned}
& E=\gamma\left(\triangle_{E \times F}\right) \cup\left\{\eta\left(0_{c_{0}}\right)\right\} \\
& F=\gamma\left(\triangle_{F \times E}\right) \cup\left\{\eta\left(0_{c_{0}}\right)\right\} .
\end{aligned}
$$

That is $E, F \subseteq[0,1]$ and hence their closures are compact. Also $\eta_{n}\left(\gamma_{n}\left(l_{n}, m_{n}\right)\right)=\eta\left(\alpha_{n} l_{n}\right)=\frac{\left(\alpha_{n}\right)^{2} l_{n}}{3}=$ $\gamma_{n}\left(\eta\left(l_{n}\right), \eta\left(m_{n}\right)\right)$

Thus by using Theorem 3.1, $\gamma$ and $\eta$ have exactly one "common coupled fixed point" in $T \times T$.

\section{Conclusion}

Firstly, we proved the common coupled fixed point theorems by using the modified definition of weakly $\mathbf{F}$-contractive mapping on a TS. After that we furnish an example based on the proved result. Our results enlarge and generalise the various results in literature.

\section{REFERENCES}

[1] D. Bugajewski.Some remarks on Kuratowski's measure of noncompactness in vector spaces with a metric. Commentationes Mathematicae 32, 5-9, (1992).

[2] D. Bugajewski. Fixed point theorems in locally convex spaces. Acta Mathematica Hungarica 94(4):345-355, (2003).

[3] D. Bugajewski and P. Kasprzak. Fixed point theorems for weakly F-contractive and strongly F-expansive mappings. Journal of Matematical Analysis and Applications 359(2009), 126134, (2009).

[4] Y. Q. Chen, Y. J. Cho, J. K. Kim and B. S. Lee. Note on KKM maps and applications. Fixed Point Theory and Applications 2006,1-9, (2006).

[5] Y. J. Cho, Guang and Y. J. Huang. The existence results of coupled quasi-solutions for a class of operator equations. Bull. korean math. Soc. 47, 455-465, (2010).

[6] Y. J. Cho, M. H. Shah and N. Hussain. Coupled fixed points of weakly F-contractive mappings in topological spaces. Applied Mathematics Letters 24(2011) 1185-1190,(2011).

[7] A. Dugundj and Granas.Fixed point theory vol.1, PWN, Warsaw, 1982, (1982).

[8] M. Furi and Vignoli. A fixed point theorem in complete metric spaces. Boll. Unione Mat. Ital. Serie IV, 505-509, (1969).

[9] X. Liu, Mi. Zhou and B. Damjanovic. Common coupled fixed Point theorem for Geraghty-type contraction in partially ordered metric spaces.Journal of Function Spaces 2018, Article ID 9063267,11 pages, (2018).

[10] M. H. Shah, N. Hussain and A. R. Khan. Common fixed points of weakly contractive and strongly expansive mappings in topological spaces. Journal of Inequalities and Applications 2010, Article ID 746045, 15 pages, (2010).

[11] H. K. Nashine and W. Shatanawi. Coupled common fixed point theorems for a pair of commuting mappings in partially ordered complete metric spaces. Computers and Mathematics with Applications, 62(4), 1984-1993, (2011). 